\title{
Spherical and Spatial Distribution of WZ Sagittae Type Dwarf Novae
}

\author{
Kenji Tanabe* \\ Okayama University of Science \\ E-mail: tanabe@big.ous.ac.jp \\ Noritoshi Ohtani \\ Graduate course of Okayama University of Science \\ E-mail: noritoshi.otani@gmail.com
}

\begin{abstract}
Nearly 100 WZ Sagittae-type dwarf novae are listed by the end of August 2015 in the AAVSO database.This type of variable stars are distributed much more isotropically than the Galactic novae.Moreover very few of them are in the direction to the Galactic center.The distance of each WZ Sge-type star can be estimated by assuming that the luminosity at maximum light is the same.Then we can depict the spatial distribution of the WZ Sge-type stars The requirement that all of these WZ Sge-type star are confined within the galactic thin disc gives a constraint on the extinction of the optical light due to the interstellar matter in the galactic thin disc
\end{abstract}

The Golden Age of Cataclysmic Variables and Related Objects - III, Golden2015

7-12 September 2015

Palermo, Italy

\footnotetext{
* Speaker.
} 


\section{Introduction}

WZ Sagittae stars are known as a subtype of dwarf novae with short (approximately 80 minutes) orbital periods and large amplitude of outburst brightness(7-8 magnitudes) compared with other type of DNe.Their photometric properties are similar to that of the SU Ursae Majoris type DNe showing superhumps during superoutbursts but exhibiting no or rare normal outbursts between superoutbursts. Moreover the time separation between superoutbursts is much longer(decades) than other SU UMa-type stars. This is thought to be due to small amount of mass transfer rate possibly at their final stage of cataclysmic variable evolution.

The prototype of these stars, WZ Sagittae, was discovered in 1919 in the archived Harvard plate[1], which recorded its outburst in 1913(subsequent outbursts are recorded in 1946,1978 and 2001)..Since then its outburst recurred every 33 year separation, as if it were a recurrent nova.However the luminosity at its maximum light is much smaller than that of the recurrent novae. By the end of the last century,only a few members are known. However recently more than 90 members are nominated in the AAVSO database. One of the reason of this is due to various automated sky survey system .Another reason is that the new stars detected in archival plates and classified as classical novae at early 20th century are now identified as WZ Sge stars. Under this circumstance, it is meaningful to consider the distribution of the WZ Sge-type stars, as this type of stars are thought to be of homogeneous sample(similar to RR Lyr stars).

This work is originated from a personal communication with Taichi Kato concerning the total number of the the WZ Sge-type star.Detailed and comprehensive analysis of this type DNe is seen in his recent paper[2].

\section{Basic data}

The catalogue of the WZ Sagittae star is created from the AAVSO database using(thanks to) the AAVSO VSX.At the end of August 2015,except WZ Sge itself,96 members are nominated. After 2005, the number is rapidly increasing. In order to make a map o the WZ Sge-type star,it is necessary for us to transform from $(\alpha, \delta)$ to Galactic coordinate $(l, b)$,by using the spherical triangle formulas,together with the software "Python".The detailed database will be published in a different paper.

\section{Spherical distribution}

After transformation from equatorial to galactic coordinates $(l, b)$,we can obtain the map in the Mollweide expression(see Fig.1). From this figure,we can see that the WZ Sge-type stars seems to be distributed rather isotropic.Moreover there exist few stars of this type at the direction to the Galactic center.This is thought to be the galactic extinction by the galactic plane similar to the effect of atmospheric extinction on the earth.On the contrary there are several stars close to the galactic north and south pole. Such a distribution like this suggests us that number density of WZ Sge-type star is homogeneous around the solar neighborhood. 


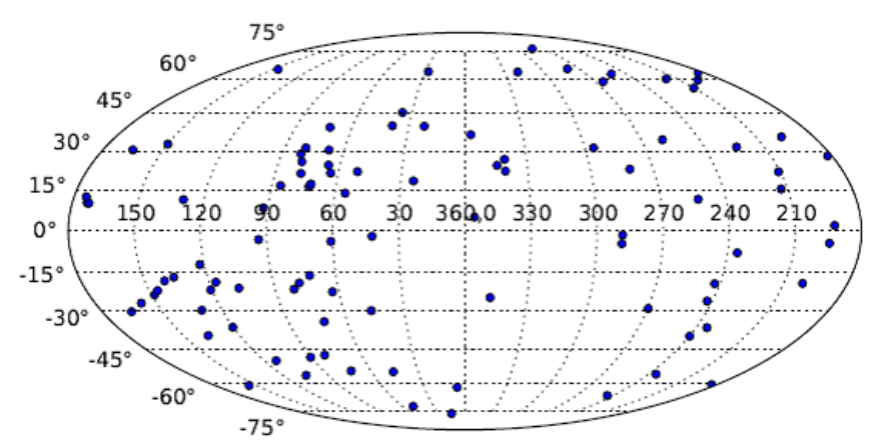

Figure 1: spherical distribution of the WZ Sge stars

Spherical disteribution of the $96 \mathrm{WZ}$ Sagittae stars is depicted in the Mollweide map,according to the AAVSO database.

\section{Spatial distribution}

As is mentioned,WZ Sge type stars are homogeneous samples because of its orbital period and amplitude of the brightness(magnitude difference). So as a working hypothesis, the luminosity at maxmum is assumed to be the same, as is similar to some kind of pulsating variables. Fortunately, the brightest and nearest one,the WZ Sge itself,of the distance is known via HIPPARCOS observation.If we take the WZ Sge's maximum light as 7.0p magnitude[1](maximum light of visual magnitude is $8.1[2])$. is, taking into account the distance $43.5 \pm 0.3 \mathrm{pc}$ [6], we can calculate the absolute magnitude as $3.8 \mathrm{mag}$ (according to Harrison[6], this value is $4.91 \mathrm{~V}$ ). Applying this value to each of the WZ Sge star,we can obtain the spatial distribution of this type ,using the following relation:

$$
\begin{gathered}
r=10^{(m+1.19) / 5} \\
X=r \cos b \cos l \\
Y=r \cos b \sin l \\
Z=r \sin b
\end{gathered}
$$

The resulting three dimensional maps are in Fig.2. As a result two OGLE DNe objects named OGLE-GD-DN-001 and DN-014 by their deep survey are far distant(6.29 Kpc and 8.29Kpc respectively) and are to be inside the Galactic thin disc. (Fig.2).

\section{Comparison with the distribution of the galactic novae}

Spherical and spatial distribution of the galactic (classical and recurrent)novae is depicted by Imamura(2013)[3],using MMRD(Maximum apparent magnitude is 15.6 magnitude versus Rate 


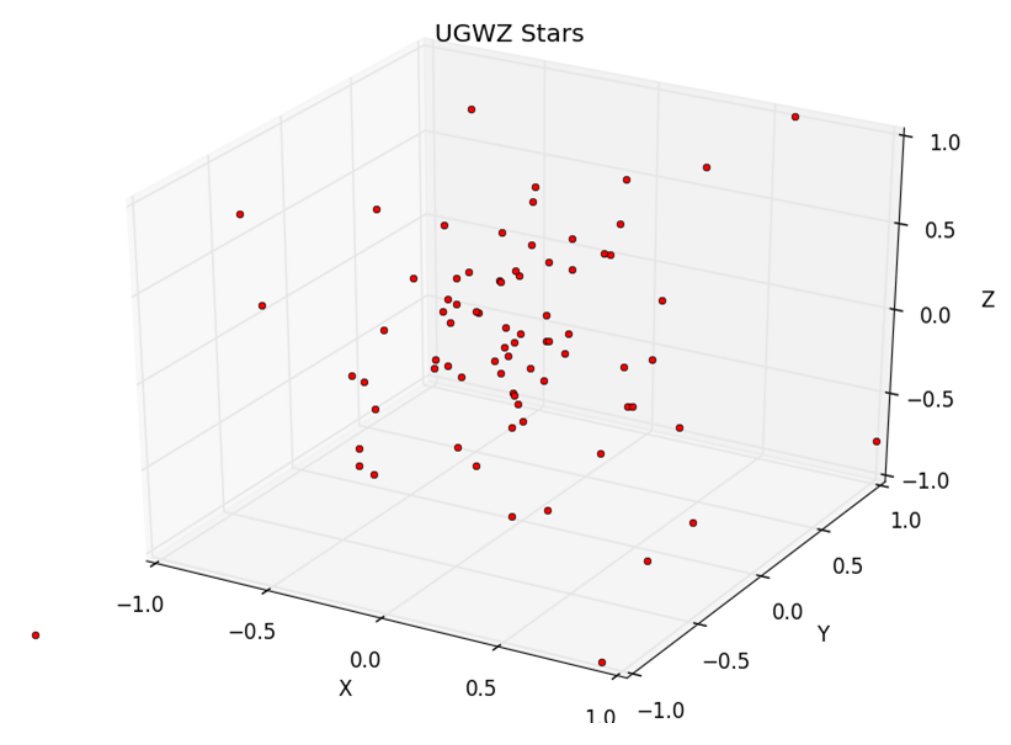

Figure 2: Spatial distribution of the WZ Sge stars

Spacial distribution of the $96 \mathrm{WZ}$ Sagittae stars is depicted by transforming from the galactic coordinate to three dimensional space $(X, Y, Z) \mathrm{Kpc}$. Two OGLE DNe are far out of 3D map .

of Decline)relation(Fig.3 and Fig.4). As is expected many of the $\mathrm{CN}$ are in the direction of the galactic center and on the concentrated on the galactic plane(except KT Eridani[3],[4]), and almost no novae are seen at high galactic latitude.In spite of large amplitude, WZ Sge star's behavior seems to attribute much lower luminosity compared with classical novae(-4 to -6 mag). Also number density of $\mathrm{CNe}$ is much smaller than WZ Sge stars.

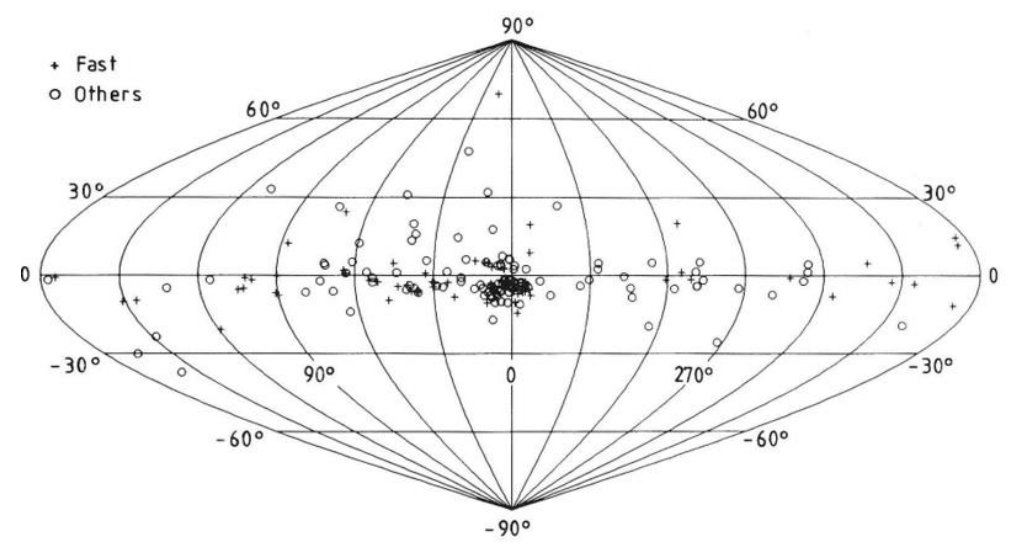

Figure 3: Spherical distribution of Galactic novae

Spherical distribution of the Galactic novae is depicted .Concentration on the Galactic plane are clearly be seen.( Adopted from Imamura(2013)).

\section{Extinction}

Without taking into account the extinction, the highest (largest Galactic hight) WZ Sge-type 


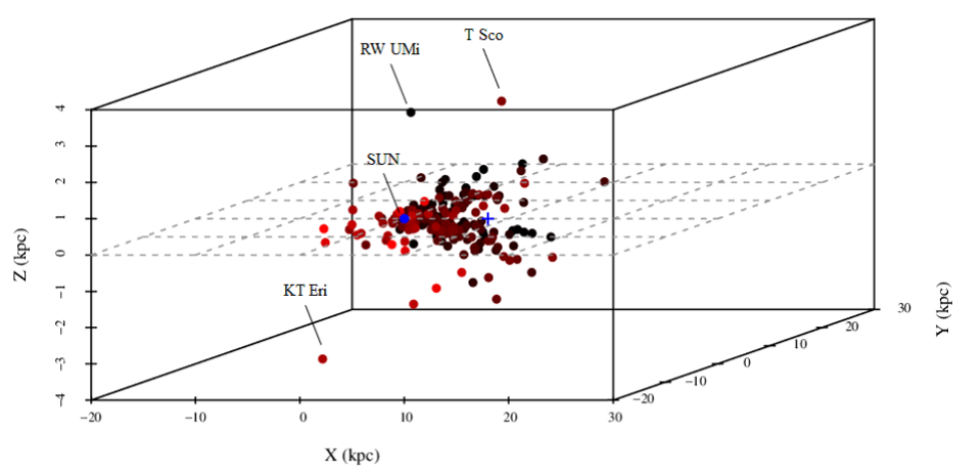

Figure 4: Spacial distribution of Galactic novae

Spacial distribution of the Galactic novae is depicted assuming their distance by MMRD. (adopted from Imamura[3]).

star SS LMi.The position of SS LMi is $(l . b)=(197.00 \mathrm{deg}, 59.86 \mathrm{deg})$.Taking into account the apparent magnitude of it is $m_{V}=15.6$,its calculated distance without extinction is $2.28 \mathrm{Kpc}$. Then its spatial position is $(X, Y, Z)=(-1.10,-0.34,+1.97) \mathrm{Kpc}$.So as to satisfy the condition that this object is to be within the Galactic thin disc, $0.35 \mathrm{Kpc}$,the extinction $A$ can be calculated by using the above mentioned values.Here $A$ is defined as follows:

$$
m-M=5 \log (r / 10)+A(l, b) .
$$

As a result,we obtain the following value

$$
A(l, b)=3.8
$$

, which is not so different (less than $1 \mathrm{mag}$ )from the value assuming the $m_{\max }=8.1 \mathrm{~V}$.

Even taking into account the extinction,2 stars of the OGLE objects [4] are as far as the distance to the galactic center.To avoid this contradiction, it is necessary to take into account the different kind of extinction not for visual but IR one .

\section{Conclusions and Discussion}

We have tried to show the spherical and spatial distribution of the WZ Sge-type dwarf novae that can be a probe to the local structure of galactic thin disc.From the above mentioned consideration, we can say the following remarks:

1. WZ Sge-type dwarf novae are distributed isotropically compared with the Galactic novae. From the tentative spatial distribution,high number density around soler system is expected.

2. Strong extinction is to be considered so as to explain the distribution of WZ Sge-type DNe inside the thin disk of Galaxy.This can explain the reason why few are detected in the direction to the Galactic center.

3. Accordinly,much more WZ Sge-type stars toward the galactic center will be detected in the future by making use of deep survey systems. 
4. If we adopt the value $V_{\max }=8.1$ [6] instead of $V_{p}=7.0$, we recalculate the value of $A$ as $A_{V}$ by using the relation

$$
A_{V}=a \cdot r
$$

Assuming that $a$ is independent of $(l, b)$ around solar neighborhood,we obtain the value of

$$
a=0.0087 \mathrm{mag} / \mathrm{pc}
$$

by solving the following algebraic equation numerically:

$$
m-M_{V}=5 \log (r / 10)+a \cdot r .
$$

$A_{V}$ of the highest $\mathrm{z}$ object SS LMi can be obtained according to this value o $a$, as

$$
A_{V}=3.01 \text {. }
$$

This value is not so different from the previous result by less than 1 magnitude.

5. In order to perform deep survey for new WZ Sge stars, one possible and simple way is to construct a automated single or multiple wide-field CCD camera pointing around the North and South Galactic pole.Such a system seems to be similar to the photographic system of early 20th century. The basic concept of this system is that WZ Sge stars are expected to be detected isotropically .

\section{References}

[1] Mackie,J.C 1919 AN 210 , 79 ;See also Duerbeck, H.(1987) A Reference Catalogue and Atlas of Galactic Novae (Reidel)

[2] Kato,T.,2015,arXiv:1507.07659v1

[3] Imamura,K. 2013,Ph.D thesis,in Japanese

[4] Imamura,K and Tanabe,K. ,2012 PASJ 64,120

[5] Mroz,P. et al.,2013,Acta Astron 63,135

[6] Harrison Th.E.. et al.,2004 AJ 127,460 